\title{
Foveal-Sparing ILM Peeling in a Case with Vitreomacular Traction and Foveal Detachment
}

\author{
Christoph Leisser Oliver Findl \\ VIROS - Vienna Institute for Research in Ocular Surgery, A Karl Landsteiner Institute, \\ Hanusch Hospital, Vienna, Austria
}

\section{Keywords}

Foveal-sparing ILM peeling · Vitreomacular traction · Foveal detachment

\begin{abstract}
A pseudophakic female patient, 80 years of age, presented with a vitreomacular traction and foveal detachment at her right eye. To avoid development of a full-thickness macular hole during surgery, foveal-sparing ILM peeling was performed. After surgery, distance-corrected visual acuity increased from 0.3 to 0.6 (Snellen) 3 months after surgery and fovea was re-attached again with restoration of the retinal layers.
\end{abstract}

\section{Introduction}

Foveal-sparing ILM peeling, proposed by Ho et al. [1] and Shimada et al. [2] in 2012, is a rather new trend in macular surgery. The aim of foveal-sparing ILM peeling is to minimize surgically induced trauma on the retinal structures. Indications with possible potential for this surgical method are epiretinal membranes (ERM), myopic foveoschisis, and full-thickness macular holes. While foveal architecture showed alterations in intraoperative optical coherence tomography in a majority of patients with myopic foveoschisis during ILM peeling including the foveal area, patients that underwent foveal-sparing ILM peeling did not show changes during surgery [3], indicating benefits regarding better anatomical results and visual acuity for foveal-sparing ILM peeling in this indication, as shown by several studies [2-4]. Furthermore, foveal-sparing ILM peeling for macular hole repair resulted in a high macularhole closure rate and better postoperative visual acuity compared to patients that underwent standard ILM peeling [5, 6]. In contrast to the above-mentioned indications for foveal-sparing 
ILM peeling, patients with foveal-sparing ILM peeling during vitrectomy for ERM removal had higher ERM recurrence rates than in cases with ILM removal including the foveal area [7]. We present a case with vitreomacular traction and foveal detachment with thinned retinal layers in the fovea that underwent vitrectomy with foveal-sparing ILM peeling.

\section{Case Report}

A pseudophakic female patient, 80 years of age, presented with a vitreomacular traction and foveal detachment with thinned retinal layers in the fovea at her right eye. To avoid development of a full thickness macular hole during surgery, 25-G pars plana vitrectomy with foveal-sparing ILM peeling, using multiple curvilinear parafoveal peels [8], and air tamponade at the end of surgery was performed. The patient performed face-down positioning for several hours after surgery. At the follow-up 1 week after surgery, distance-corrected visual acuity increased from 0.3 to 0.5 (Snellen), fovea was re-attached again, and retinal layers showed restoration with improved foveal thickness (Fig. 1). Distance-corrected visual acuity further increased to 0.6 (Snellen) at the follow-up 3 months after surgery.

\section{Discussion}

Surgical removal of the ILM seems reasonable in cases with foveal detachment, as beside vitreomacular traction, it was reported that tangential traction of the ILM, a further possible reason for foveal detachment, has the potential to induce impending macular holes [9]. In our case, thinning of the retinal layers at the fovea raised concerns regarding ILM peeling including the foveal area, as there would have been an increased risk of intraoperative development of a full-thickness macular hole.

Foveal-sparing ILM peeling, already described for other indications, was shown to have potential to be beneficial for patients with myopic foveoschisis [1-4] and full-thickness macular hole $[5,6]$, as described above. In contrast, foveal-sparing ILM peeling for removal
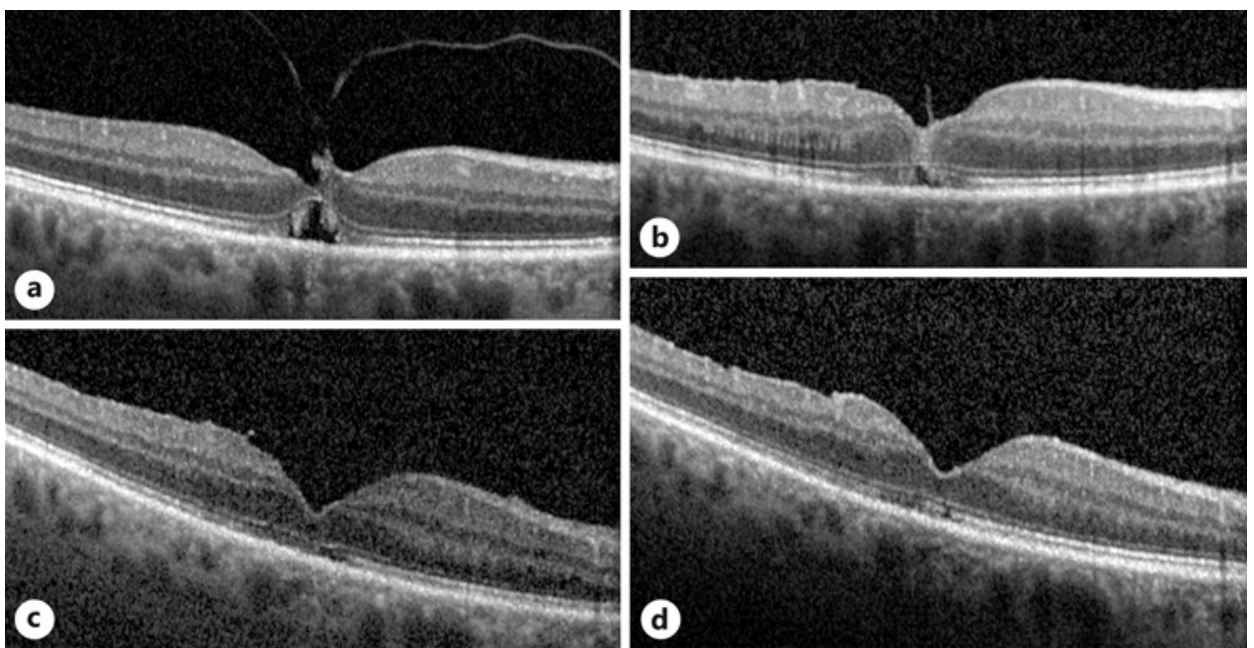

Fig. 1. A patient with vitreomacular traction, foveal detachment, and disruption of the superficial foveal layers (a) that underwent 25-G pars plana vitrectomy with foveal-sparing ILM peeling showed re-attachment of the fovea and gliotic scarring at the area of tissue restoration on the foveal surface 1 day after surgery (b), and restoration of the retinal layers 1 week (c) and 3 months after surgery (d). 
of ERM led to more recurrences of ERM in the postoperative time course [7]. Due to the disrupted retinal surface with retinal thinning in our patient (Fig. 1a), concerns to induce development of a full-thickness macular hole during complete ILM peeling were higher than the risk of postsurgical ERM development in the foveal area.

Pneumatic release with intravitreal injection of a gas bubble would have been an alternative surgical approach for release of the vitreomacular traction $[10,11]$, but with the risk of development of a full-thickness macular hole or a retinal detachment [10,11]. Furthermore, as this procedure does not treat tangential traction of the ILM at the retinal surface, there would have been a risk of postoperative development of full-thickness macular hole despite successful pneumatic release in our patient. Concluding, foveal-sparing ILM peeling showed to be a safe and effective surgical option in our patient with vitreomacular traction and foveal detachment with preoperative thinning of the retinal layers.

\section{Statement of Ethics}

Written informed consent was obtained from the patient to publish the case.

\section{Conflict of Interest Statement}

O. Findl is a scientific advisor for Alcon, Croma, Carl Zeiss Meditec AG, and Merck. All authors declare that there are no conflicts of interest.

\section{Funding Sources}

There was no funding.

\section{Author Contributions}

C. Leisser and O. Findl: conception of the study, data acquisition, preparation of the manuscript, and critical review of the manuscript.

\section{References}

1 Ho TC, Chen MS, Huang JS, Shih YF, Ho H, Huang YH. Foveola nonpeeling technique in internal limiting membrane peeling of myopic foveoschisis surgery. Retina. 2012;32(3):631-4.

2 Shimada N, Sugamoto Y, Ogawa M, Takase H, Ohno-Matsui K. Fovea-sparing internal limiting membrane peeling for myopic traction maculopathy. Am J Ophthalmol. 2012;154(4):693-701.

3 Itoh Y, Inoue M, Kato Y, Koto T, Hirakata A. Alterations of foveal architecture during vitrectomy for myopic retinoschisis identified by intraoperative optical coherence tomography. Ophthalmologica. 2019;242(2): 87-97.

4 Ho TC, Yang CM, Huang JS, Yang CH, Yeh PT, Chen TC, et al. Long-term outcome of foveolar internal limiting membrane nonpeeling for myopic traction maculopathy. Retina. 2014;34(9):1833-40.

5 Ho TC, Yang CM, Huang JS, Yang CH, Chen MS. Foveola nonpeeling internal limiting membrane surgery to prevent inner retinal damages in early stage 2 idiopathic macula hole. Graefes Arch Clin Exp Ophthalmol. 2014;252(10):1553-60.

6 Murphy DC, Fostier W, Rees J, Steel DH. Foveal sparing internal limiting membrane peeling for idiopathic macular holes: effects on anatomical restoration of the fovea and visual function. Retina. 2020;40(11):212733. 
7 Russo A, Morescalchi F, Gambicorti E, Cancarini A, Costagliola C, Semeraro F. Epiretinal membrane removal with foveal-sparing internal limiting membrane peeling: a pilot study. Retina. 2019;39(11):2116-24.

8 Jin H, Zhang Q, Zhao P. Fovea sparing internal limiting membrane peeling using multiple parafoveal curvilinear peels for myopic foveoschisis: technique and outcome. BMC Ophthalmol. 2016;16(1):180.

9 Bentaleb Machkour Z, Garweg JG, Bandello F, Denis P, Kodijkian L. Differences in the presentation of stage 1 macular holes illustration by optical coherence tomography. Eur J Ophthalmol. 2020.

10 Claus MG, Feron E, Veckeneer M. Pneumatic release of focal vitreomacular traction. Eye. 2017;31(3):411-6.

11 Chan CK, Crosson JN, Mein CE, Daher N. Pneumatic vitreolysis for relief of vitreomacular traction. Retina. 2017; 37(10):1820-31. 